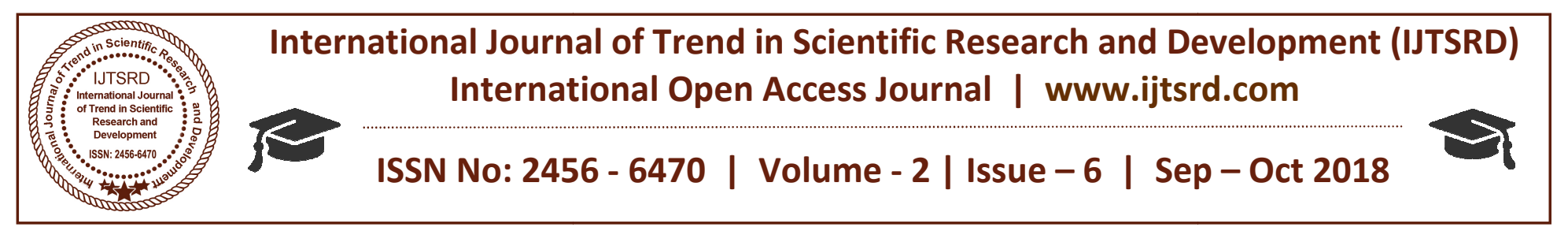

\title{
Design of a Controller for Systems with Simultaneously Variable Parameters
}

\author{
Prof. Kamen Yanev \\ Department of Electrical Engineering, Faculty of Engineering and Technology, Gaborone, Botswana
}

\begin{abstract}
The contribution of this paper is in suggesting an analysis and design of a control system with variable parameters. By applying the recommended by the author method of the Advanced D-partitioning the system's stability can be analyzed in details. The method defines regions of stability in the space of the system's parameters. The designed controller is enforcing desired system performance. The suggested technique for analysis and design is essential and beneficial for the further development of control theory in this area.
\end{abstract}

Keyword: System with variable parameters, Stability regions, System performance

\section{INTRODUCTION}

Control systems performance must be insensitive to parameter variations. In the process of design such a control system, it is important to determine the regions stability, related to the variation of the system parameters. The suggested by the author method, dealing with the effects of parameters variations on the system's stability, is classified as Advanced Dpartitioning [1], [2], [3]. It is an efficient tool for system stability analysis in case of variation of any of the system's parameters. It is further upgraded in this paper and can be used for simultaneously varying parameters. This research is also suggesting a method for design of a controller, by applying forward-series compensation. It can suppress the influence of any parameters variations of the control system. Innovation is demonstrated in the unique property of the designed controller that can operate effectively for variations of any one of the system's parameters within prescribed limits. The design of the controller is based on the ITAE criterion [4], [5]. For higher order systems, a pair of dominant poles represents the system dynamics. The relative damping ratio $\zeta$ of the system is taken as a performance objective for the optimization design.

\section{System with Simultaneously Variable Parameters}

The Advanced D-Partitioning analysis in case of two simultaneously variable parameters [2], [4], [6] can be demonstrated for a control system of the armaturecontrolled dc motor and a type-driving mechanism. The gain and one of the time-constants are uncertain and variable. The open-loop transfer function of the system can be presented as:

$$
G_{P O}(s)=\frac{K}{(1+T s)(1+0.5 s)(1+0.8 s)}
$$

The characteristic equation of the unity feedback system is derived as:

$K+(1+T s)(1+0.5 s)(1+0.8 s)=0$

By substituting $s=j \omega$ equation (2) is modified to:

$K=-1+(1.3 T+0.4) \omega^{2}+j \omega\left(0.4 T \omega^{2}-1.3-T\right)$

Since the gain may have only real values, the imaginary term of equation (3) is set to zero. Then:

$\omega^{2}=\frac{1.3+T}{0.4 T}$

The result of (4) is substituted into the real part of equation (3), from where:

$$
K=\frac{1.3 T^{2}+1.69 T+0.52}{0.4 T}=3.25 T+4.225+\frac{1.3}{T}
$$

The D-Partitioning curve $K=f(T)$ is plotted with the aid of the following code:

$$
\begin{aligned}
>>\mathrm{T} & =0: 0.1: 5 ; \\
>>\mathrm{K} & =3.25 . * \mathrm{~T}+4.225+1.3 . / \mathrm{T} \\
\mathrm{K} & =
\end{aligned}
$$


Columns 1 through 10

$\begin{array}{lllllll}\text { Inf } & 17.5500 & 11.3750 & 9.5333 & 8.7750 & 8.4500 \\ 8.3417 & 8.3571 & \mathbf{8 . 4 5 0 0} & \mathbf{8 . 5 9 4 4} & & \end{array}$

Columns 11 through 20

$\begin{array}{ccccccc}8.7750 & 8.9818 & 9.2083 & 9.4500 & 9.7036 & 9.9667 \\ 10.2375 & 10.5147 & 10.7972 & 11.0842 & & \end{array}$

Columns 21 through 30
11.3750
11.6690
11.9659
$12.2652 \quad 12.5667$
$\begin{array}{lllll}12.8700 & 13.1750 & 13.4815 & 13.7893 & 14.0983\end{array}$

Columns 31 through 40

\section{$\begin{array}{lllll}14.4083 & 14.7194 & 15.0313 & 15.3439 & 15.6574\end{array}$} $\begin{array}{lllll}15.9714 & 16.2861 & 16.6014 & 16.9171 & 17.2333\end{array}$

Columns 41 through 50
$17.5500 \quad 17.8671$
18.1845
18.5023
18.8205

19.138919 .4576

\subsection{6}

20.095820 .4153

\section{Column 51}

20.7350

>> Plot $(\mathrm{T}, \mathrm{K})$

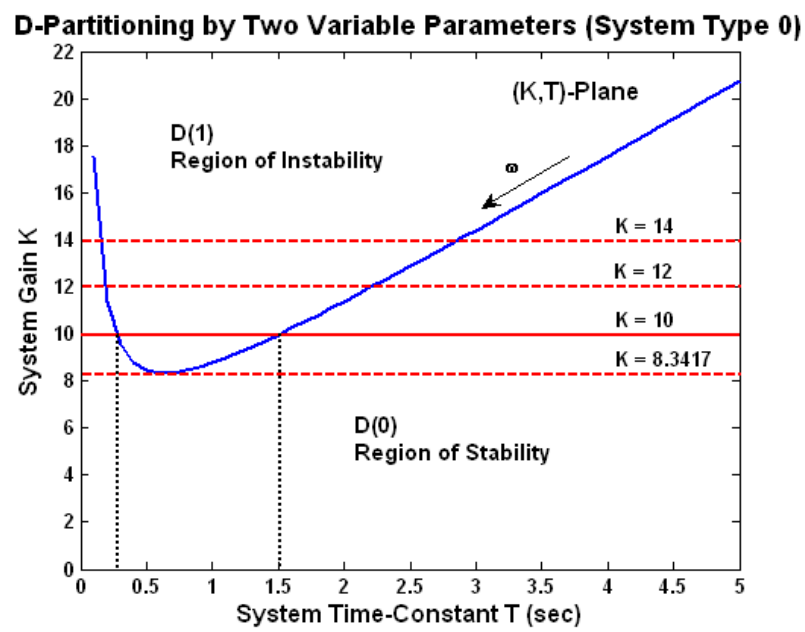

Fig.1. Advanced D-Partitioning in terms of two variable parameters

The D-Partitioning curve $K=f(T)$ defines the border between the region of stability D (0) and instability $\mathrm{D}(1)$ for case of simultaneous variation of the two system parameters. Each point of the DPartitioning curve represents the marginal values of the two simultaneously variable parameters. This is a unique advancement and an innovation in the theory of control systems stability analysis. The demonstration of the system performance in case of variation of the time-constant $T$ is done at gain set to $K=10$. When $0<T<0.25 \mathrm{sec}$ and $T>1.5 \mathrm{sec}$ the system is stable. But it becomes unstable in the range $0.25 \mathrm{sec}<T<1.5 \mathrm{sec}$. It is also obvious that the system performance and stability depends on the interaction between the two simultaneously varying parameters. If $K<8.3417$, the system is stable for any value of the $T$. Higher values of $K(K=12, K=$ 14), enlarge the range of $T$ at which the system will fall into instability.

III. Design of a Controller for Systems with Simultaneously Variable Parameters

The open-loop transfer function of the plant $G_{P O}(s)$ is modified and now presented in equation (6) considering the two variable parameters that are the system's gain $K$ and time-constant $T$. Initially, it is suggested that the gain is set to $K=10$, while $T$ is variable [5], [7], [8].

$$
\begin{aligned}
G_{P O}(s) & =\frac{K}{(1+T s)(1+0.5 s)(1+0.8 s)}= \\
& =\frac{K}{0.4 T s^{3}+(1.3 T+0.4) s^{2}+(1.3+T) s+1}
\end{aligned}
$$

The robust controller consists of a series stage $G_{S 0}(\mathrm{~s})$ and a forward stage $G_{F O}(\mathrm{~s})$. An integrating stage $G_{I O}(\mathrm{~s})$ is also included in the controller as seen from Figure 2.

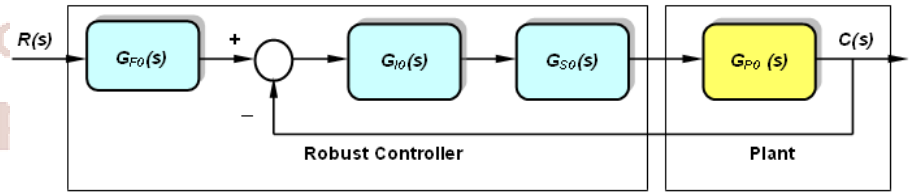

Fig.2. Robust controller incorporated into the control system

Initially, the plant transfer function $G_{P O}(\mathrm{~s})$, as a standalone block, is involved in a unity feedback system having a closed-loop transfer function presented as:

$$
\begin{aligned}
G_{C L}(s) & =\frac{K}{(1+T s)(1+0.5 s)(1+0.8 s)+K}= \\
& =\frac{K}{0.4 T s^{3}+(1.3 T+0.4) s^{2}+(1.3+T) s+1+K}
\end{aligned}
$$

Equation (7) is used as a base in the design strategy for constructing the series stage of the robust controller. It has the task to place its two zeros near the desired dominant closed-loop poles, that satisfy the condition $\zeta=0.707$ [9], [10]. These zeros will become the dominant poles of the unity negative feedback system, involving the cascade connection of the series controller stage $G_{S O}(s)$, the integrator $G_{I O}(s)$ 
and the plant $G_{P O}(s)$. If initially the gain is set to $K=$ 10, the optimal value of the time-constant $T$, corresponding to the relative damping ratio $\zeta=0,707$ of the closed-loop system, is determined by the code:

\section{$>\mathbf{T}=[20: 0.01: 35]$;}

$>>$ For $n=1$ : length $(\mathrm{T})$

G_array $(:,:, n)=\operatorname{tf}([10],[0.4 * T(n)(1.3 * T(n)+0.4)$

$(1.3+T(n)) 11]) ;$ end

$>[\mathbf{y}, \mathrm{z}]=$ damp $\left(\mathbf{G}_{-}\right.$array $)$;

$>>\mathbf{y}, \mathbf{z}]=$ damp $\left(G_{-}\right.$array $)$;

$>$ Plot $(\mathrm{T}, \mathrm{z}(1, \mathbf{1}))$

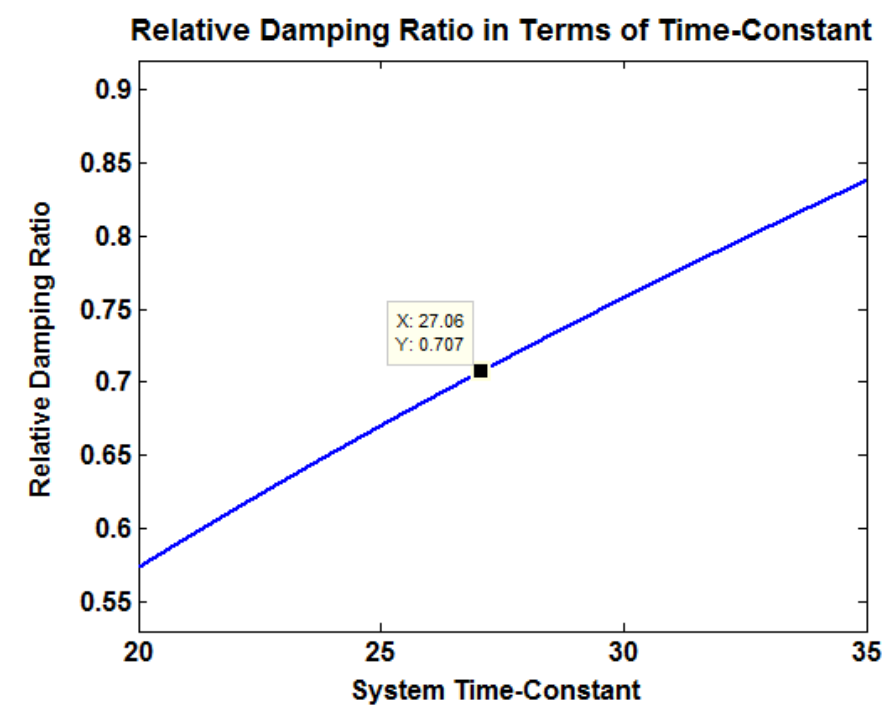

Figure3. Time-constant corresponding to $\zeta=0,707$

As seen from Figure 3, the relative damping ratio is $\zeta=0.707$ when the time-constant is $T=27.06 \mathrm{sec}$.

By substituting the values $T=27.06 \mathrm{sec}$ and $K=10 \mathrm{in}$ equation (7), the transfer function of the closed-loop system becomes:

$$
G_{C L}(s)=\frac{10}{10.824 s^{3}+35.578 s^{2}+28.36 s+11}
$$

The assessment of the system proves that the relative damping ratio becomes $\zeta=0.707$, when the timeconstant is $T=27.06 \mathrm{sec}$, resulting in system's desired closed-loop poles $-0.466 \pm \mathrm{j} 0.466$. These outcomes are determined from the code:

> $G C L 0=t f([10],[10.82435 .57828 .3611])$ $>\operatorname{damp}(\mathrm{GCLO})$

Eigenvalue

Damping Freq. (rad/s)

$-4.64 e-001+4.64 e-001 i$

$-4.64 e-001-4.64 e-001 i$

$-2.36 e+000$ 7.07e-001

$7.07 e-001$

$1.00 e+000$
$6.56 e-001$

$6.56 e-001$

$2.36 e+000$
The series robust controller zeros can be placed at the approximated values $-0.5 \pm \mathrm{j} 0.5$. Therefore, the transfer function of the series robust controller $G_{S O}(s)$ is:

$$
\begin{aligned}
G_{S 0}(s) & =\frac{(s+0.5+j 0.5)(s+0.5-j 0.5)}{0.5}= \\
& =\frac{s^{2}+s+0.5}{0.5}
\end{aligned}
$$

An integrating stage $G_{I 0}(\mathrm{~s})$ is added to eliminate the steady-state error of the system. It is connected in cascade with the series controller. Then, the transfer function of the compensated open-loop control system will be as follows:

$$
\begin{aligned}
G_{O L}(s) & =G_{I 0}(s) G_{S 0}(s) G_{P 0}(s)= \\
7 & =\frac{K\left(s^{2}+s+0.5\right)}{0.5 s(1+T s)(1+0.5 s)(1+0.8 s)}
\end{aligned}
$$

When $G_{O L}(\mathrm{~s})$ is involved in a unity feedback, its closed-loop transfer function is determined as:

$$
\begin{aligned}
G_{C L}(s) & =\frac{K\left(s^{2}+s+0.5\right)}{\left[\begin{array}{l}
0.5 s(1+T s)(1+0.5 s)(1+0.8 s)+ \\
+K\left(s^{2}+s+0.5\right)
\end{array}\right]}
\end{aligned}
$$

It is seen from the equation (11) that the closed-loop zeros will attempt to cancel the closed loop poles of the system, being in their area. This problem can be avoided if a forward controller $G_{F 0}(\mathrm{~s})$ is added to the closed-loop system, as shown in Figure 2. The poles of $G_{F O}(\mathrm{~s})$ are designed to cancel the zeros of the closed-loop transfer function $G_{C L}(\mathrm{~s})$, as shown in equation (12):

$$
G_{F 0}(s)=\frac{0.5}{s^{2}+s+0.5}
$$

Finally, the transfer function of the total compensated system is derived considering the diagram in Figure 2.

$$
\begin{aligned}
& G_{T 0}(s)=G_{F 0} G_{C L 0 S}(s)= \\
& =\frac{K}{\left[\begin{array}{l}
0.2 T s^{4}+(0.65 T+0.2) s^{3}+ \\
+(0.65+0.5 T) s^{2}+0.5 s+K\left(s^{2}+s+0.5\right)
\end{array}\right]}
\end{aligned}
$$

\section{Performance of the Compensated System}

The system is tested for insensitivity to variations of its gain $K$ and its time-constant $T$. Comparison of its performance is done before and after applying the 
robust compensation. Initially at system gain is set to $K=10$ and three different values of the time-constants are set successively to $T=0.1 \mathrm{sec}, T=0.8 \mathrm{sec}$ and $T=$ $2 \mathrm{sec}$ and are substituted in equation (6).

The case of $T=0.8 \mathrm{sec}$, corresponds to the region $\mathrm{D}(2)$ and definitely to an unstable control system. The cases of $T=0.1 \mathrm{sec}$ and $T=2 \mathrm{sec}$, reflect regions D1(0) and D2(0) accordingly and are related to a stable control system.

The transient responses of the system before applying the robust compensation are illustrated in Figure 4 and Figure 5 and are achieved by the following codes:

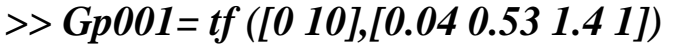

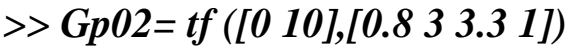

>> Gp0fb001= feedback $(G p 001,1)$

> Gp0fb02= feedback $(G p 02,1)$

> Step (Gp0fb001, Gp0fb02)

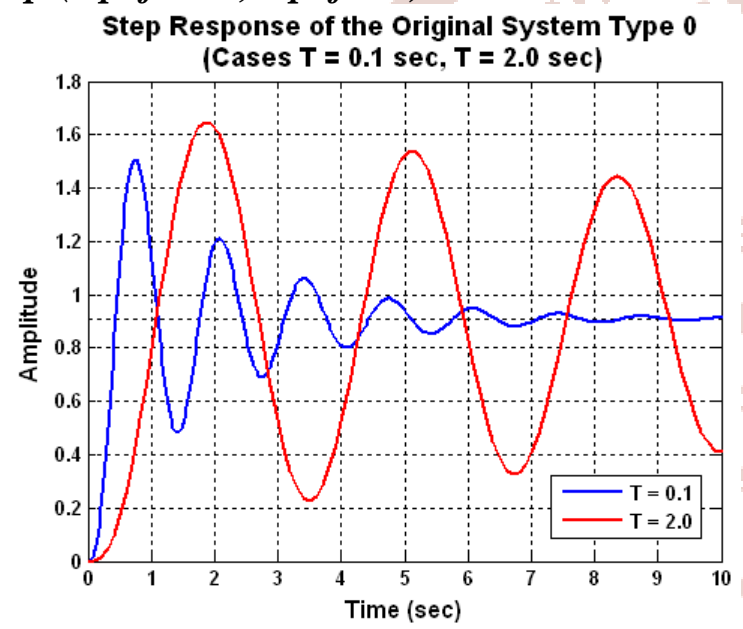

Fig.4. Step responses of the original control system $(\mathrm{T}=0.1 \mathrm{sec}, \mathrm{T}=2 \mathrm{sec}$ at $\mathrm{K}=10)$

\section{$>$ > Gp008=tf([0 10],[0.32 1.44 2.1 1]) \\ > Gp0fb008=feedback $($ Gp001,1) \\ > step (Gp0fb008)}

Step Response of the Original System Type 0

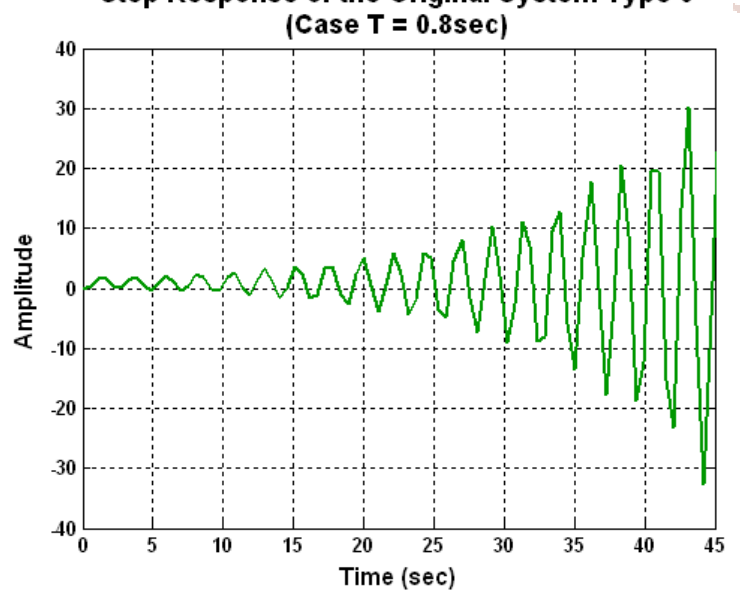

Fig.5. Step responses of the original control system $(\mathrm{T}=0.8 \mathrm{sec}$, at $\mathrm{K}=10)$
The compensated system is also examined for robustness in the time-domain. Considering the same values of the time-constant, as those used for the assessment of the original system, $T=0.1 \mathrm{sec}, T=0.8$ sec and $T=2 \mathrm{sec}$ at system gain $K=10$ and substituting them in equation (13), the following transfer functions are obtained:

$$
\begin{aligned}
& G_{T 0}(s)_{T=0.1}=\frac{10}{0.04 s^{4}+0.53 s^{3}+21.4 s^{2}+21 s+10} \\
& G_{T 0}(s)_{T=0.8}=\frac{10}{0.32 s^{4}+1.44 s^{3}+22.1 s^{2}+21 s+10} \\
& G_{T 0}(s)_{T=2}=\frac{10}{0.8 s^{4}+3 s^{3}+23.3 s^{2}+21 s+10}
\end{aligned}
$$

The step responses, representing the time-constant variation of the robust system, shown in Figure 6, are determined by the code:

$$
\begin{aligned}
& >>\text { GT01=tf([10], [0.04 0.53 21.4 21 10]) } \\
& >\operatorname{GT08}=t f([10],[0.321 .4422 .121 \quad 10]) \\
& \gg G T 20=t f\left([10],\left[\begin{array}{lllll}
0.8 & 3 & 23.3 & 21 & 10
\end{array}\right]\right) \\
& \text { >> step (GT01, GT08, GT20) }
\end{aligned}
$$

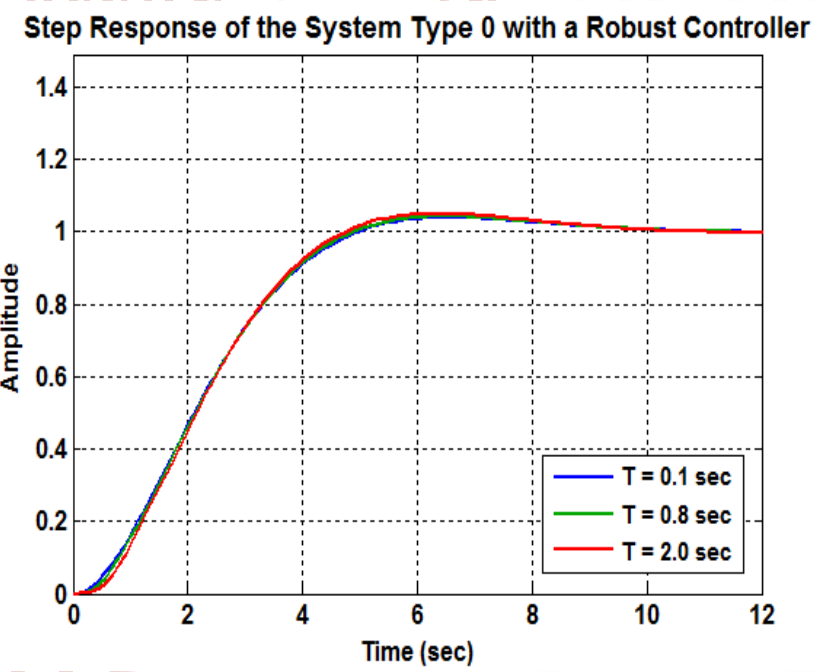

Fig.6. Step responses of the system with a robust controller $(\mathrm{T}=0.1 \mathrm{sec}, \mathrm{T}=0.8 \mathrm{sec}, \mathrm{T}=2 \mathrm{sec}$ at $\mathrm{K}=$ 10)

As seen from Figure 6, due to the applied robust controller, the control system becomes quite insensitive to variation of the time-constant $T$. The step responses for $T=0.1 \mathrm{sec}, T=0.8 \mathrm{sec}$ and $T=$ 2 sec coincide.

Since the system is with two variable parameters, now the gain will be changed, applying: $K=5, K=10, K=$ 20 , while keeping the system's time-constant at $T=$ $0.8 \mathrm{sec}$. For comparison of the system's insensitivity 
to the gain variation before and after applying the robust compensation, initially the suggested values as shown above are substituted in equation (6). The transient responses of the original system are illustrated in Figure 7 and 8 and are achieved by the following codes:

$>\operatorname{Gp05}=t f\left(\left[\begin{array}{ll}0 & 5\end{array}\right],\left[\begin{array}{llll}0.32 & 1.44 & 2.1 & 1\end{array}\right]\right)$

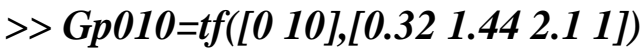

> Gp0fb5=feedback $(G p 05,1)$

$>$ Gp0fb10=feedback $(G p 010,1)$

> Step (Gp0fb5, Gp0fb10)

>> Step (Gp0fb5, Gp0fb10)

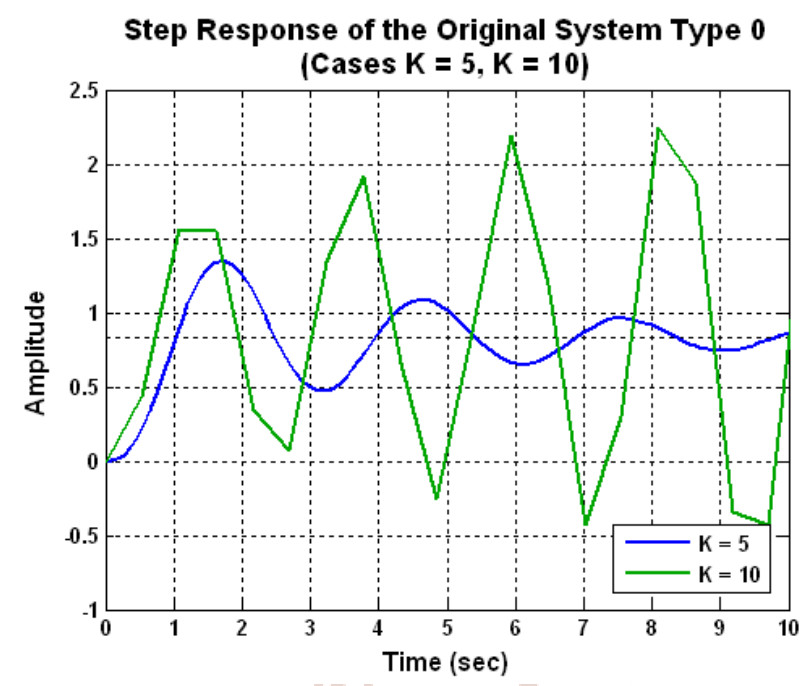

Fig.7. Step responses of the original control system $(\mathrm{K}=5, \mathrm{~K}=10$ at $\mathrm{T}=0.8 \mathrm{sec})$

>> $G p 020=t f\left(\left[\begin{array}{llll}0 & 20\end{array}\right],[0.321 .442 .11]\right)$

$>>$ Gp0fb5=feedback $(G p 05,1)$

>> Gp0fb10=feedback $(G p 010,1)$

> Gp0fb20=feedback $(G p 020,1)$

> step (Gp0fb20)

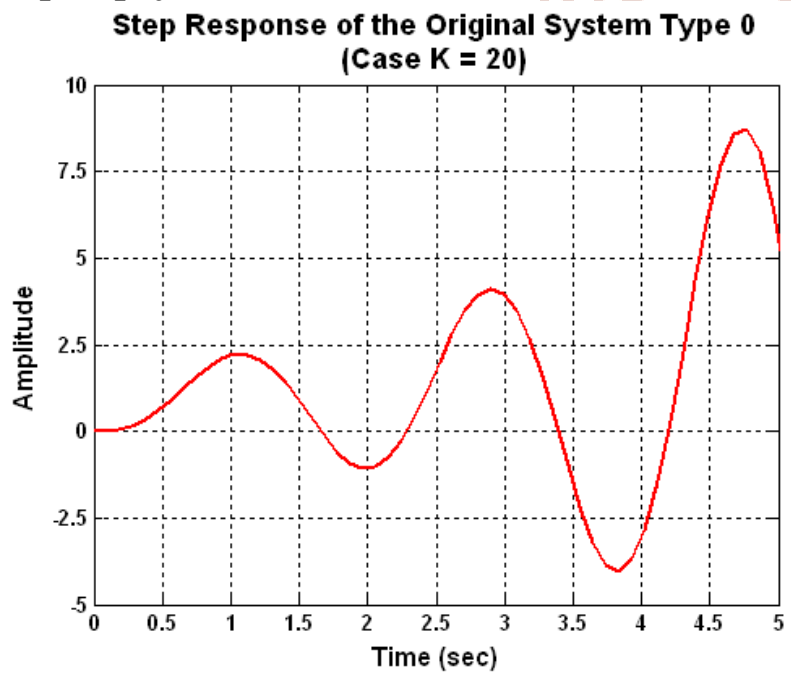

Fig.8. Step response of the original control system $(\mathrm{K}=20$ at $\mathrm{T}=0.8 \mathrm{sec})$
It is obvious that the cases of $K=10$ and $K=20$, correspond to an unstable original control system.

Next, the variable gain $K=5, K=10, K=20$ will be applying to the robust compensated system, keeping the system's time-constant at $T=0.8 \mathrm{sec}$. These values are substituted in equation (13). As a result, the following outcomes are delivered:

$$
\begin{aligned}
& G_{T 0}(s)_{K=5}=\frac{5}{0.32 s^{4}+1.44 s^{3}+12.1 s^{2}+11 s+5} \\
& G_{T 0}(s)_{K=10}=\frac{10}{0.32 s^{4}+1.44 s^{3}+22.1 s^{2}+21 s+10} \\
& G_{T 0}(s)_{K=20}=\frac{20}{0.32 s^{4}+1.44 s^{3}+42.1 s^{2}+41 s+20}
\end{aligned}
$$

To compare the system robustness before and after the robust compensation, the step responses for the three different cases, representing the gain variation of the robust system, are plotted in Figure 9 with the aid of the code as shown below:
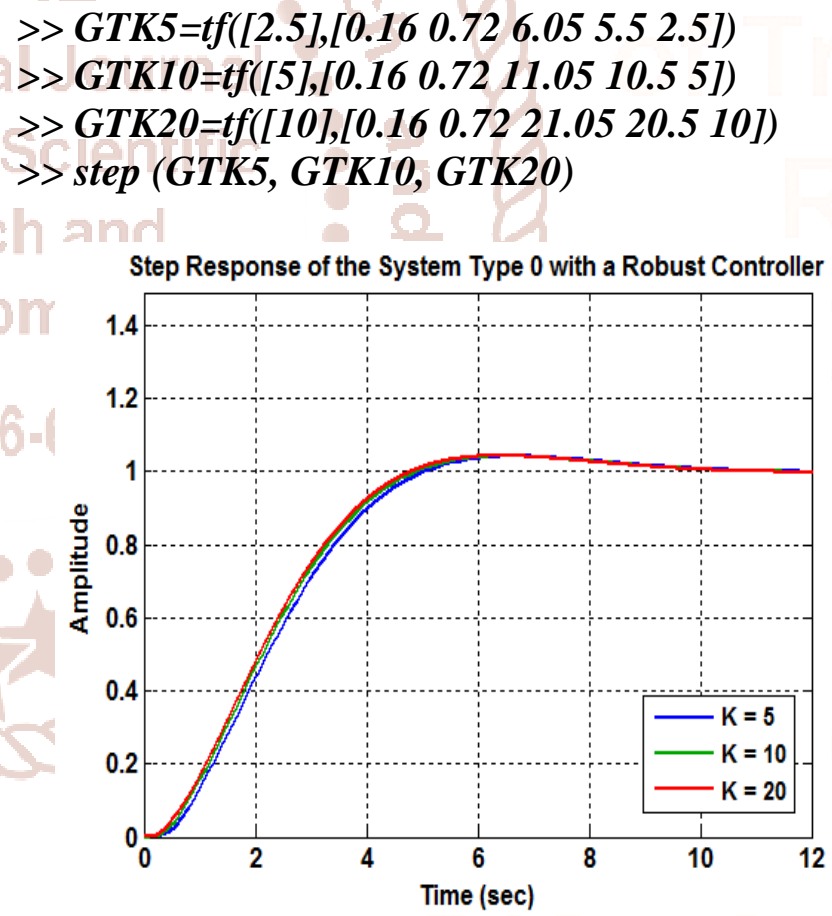

Figure9. System's step responses with the robust controller $(K=5, K=10, K=20$ at $\mathrm{T}=0.8 \mathrm{sec})$

Again, the system became robust. An additional zero included into the series robust controller stage can further improve the rise time of the system's step response.

An average case is chosen with $K=10$ and $T=0.8$ sec for the assessment of the system's performance after the application of the robust controller. This case 
will differ insignificantly from the other cases of the discussed variable $K$ and $T$. The performance evaluation is achieved by following code:

> GT10=tf $\left([10],\left[\begin{array}{llll}0.32 & 1.4422 .121 & 10\end{array}\right)\right.$
> $\operatorname{damp}(G T 10)$
Eigen value

$-4.91 e-001-4.89 e-001 i$

$-1.76 e+000+7.88 e+000 i$

$-1.76 e+000-7.88 e+000 i$
$-4.91 e-001+4.89 e-001 i$
Damping

$7.09 e-001$

$7.09 e-001$

$2.18 e-001$

$2.18 e-001$
Freq. $(\mathrm{rad} / \mathrm{s})$

$6.93 e-001$

$6.93 e-001$

$8.07 e+000$

$8.07 e+000$
It is seen that the relative damping ratio enforced by the system's dominant poles is $\zeta=0.709$, being very close to the objective value of $\zeta=0.707$. This insignificant difference is due to the rounding of the desired system's poles to $-0.5 \pm \mathrm{j} 0.5$, during the design of the series robust controller stage.

\section{Conclusions}

The D-Partitioning analysis is further advanced for systems with multivariable parameters [8], [9], [10]. The Advanced D-partitioning in case two variable parameters is demonstrating the strong interaction between the variable parameters. Each point of the DPartitioning curve represents the marginal values of the two simultaneously variable parameters, being a unique advancement and an innovation in the theory of control systems stability analysis.

The design strategy of a robust controller for linear control systems proves that by implementing desired dominant system poles, the controller enforces the required relative damping ratio and system performance. For systems Type 0 , an additional integrating stage ensures a steady-state error equal to zero.

The designed robust controller brings the system to a state of insensitivity to the variation of its parameters within specific limits of the parameter variations. The experiments with variation of different parameters show only insignificant difference in performance for the different system conditions [10], [11], [12].

For the discussed case, the system becomes quite insensitive to variation of the time constant within the limits $0.1 T<T<10 T$. The system is quite insensitive to variations of the gain $K$ within the limits $0.5 K<K$ $<5 K$. Insignificant step response difference is observed also if the experiment is repeated with different variation of the gain and different variation time-constants values.

Since the design of the robust controller is based on the desired system performance in terms of relative damping, its contribution and its unique property is that it can operate effectively for any of the system's parameter variations or simultaneous variation of a number of parameters. This property is demonstrated by the comparison of the system's performance before and after the application of the robust controller. Tests demonstrate that the system performance in terms of damping, stability and time response remains robust and insensitive in case of any simultaneous variations of the gain and the time-constant within specific limits. The suggested analysis and design is beneficial for further advancement of control theory in this field.

\section{References}

1. Yanev K., Advanced D-Partitioning Stability Analysis of Digital Control Systems with Multivariable Parameters, ICGST, Journal of Automatic Control and System Engineering, Delaware, USA, Volume 17(2), pp. 9-19, December 2017.

2. Yanev K., D-Partitioning Analysis of Digital Control Systems by Applying the Bilinear Tustin Approximation, International Review of Automatic Control (IREACO), Italy, Vol. 7, (6), pp. 517-523, November 2014.

3. - Yanev K, Advanced D-Partitioning Analysis and Comparison with the Kharitonov's Theorem Assessment, Journal of Multidisciplinary Engineering Science and Technology (JMEST), Germany, Vol. 2 (1), pp. 338-344, January 2015.

4. Shinners S., Modern Control System Theory, Addison-Wesley Publishing Company, pp.369472, 2004.

5. Driels M., Linear control System Engineering, McGraw-Hill International Inc., pp.145-222, 2006.

6. Golten J., Verwer A., Control System Design and Simulation, McGraw-Hill International Inc., pp. 278-335, 2001.

7. Bhanot S., Process Control Principles and Applications, Oxford University Press, pp. 170187, 2010.

8. Yanev K., Anderson G. O, Masupe S., Multivariable system's parameters interaction and 
International Journal of Trend in Scientific Research and Development (IJTSRD) ISSN: 2456-6470

robust control design, Journal of International Review of Automatic Control, Vol. 4(2), pp.180190, March 2011.

9. Behera L., Kar I., Intelligent Systems and Control Principles and Applications, Oxford University Press, pp. 20-83, 2009.

10. Yanev K. M., Design and Analysis of a Robust Accurate Speed Control System by Applying a Digital Compensator, ICGST Journal of Automatic Control and System Engineering,
Delaware, USA, Vol. 16(1), pp. 27-36, April 2016.

11. Megretski A., Multivariable Control systems, Proceedings of Massachusetts Institute of Technology, Vol.7 (4), 2010, pp.182-189, 2010.

12. Yanev K. M., Digital Optimal Compensation of Process Control Systems, ICGST, Journal of Automatic Control and System Engineering, Delaware, USA, Vol. 17(1), pp. 7-18, June 2017.

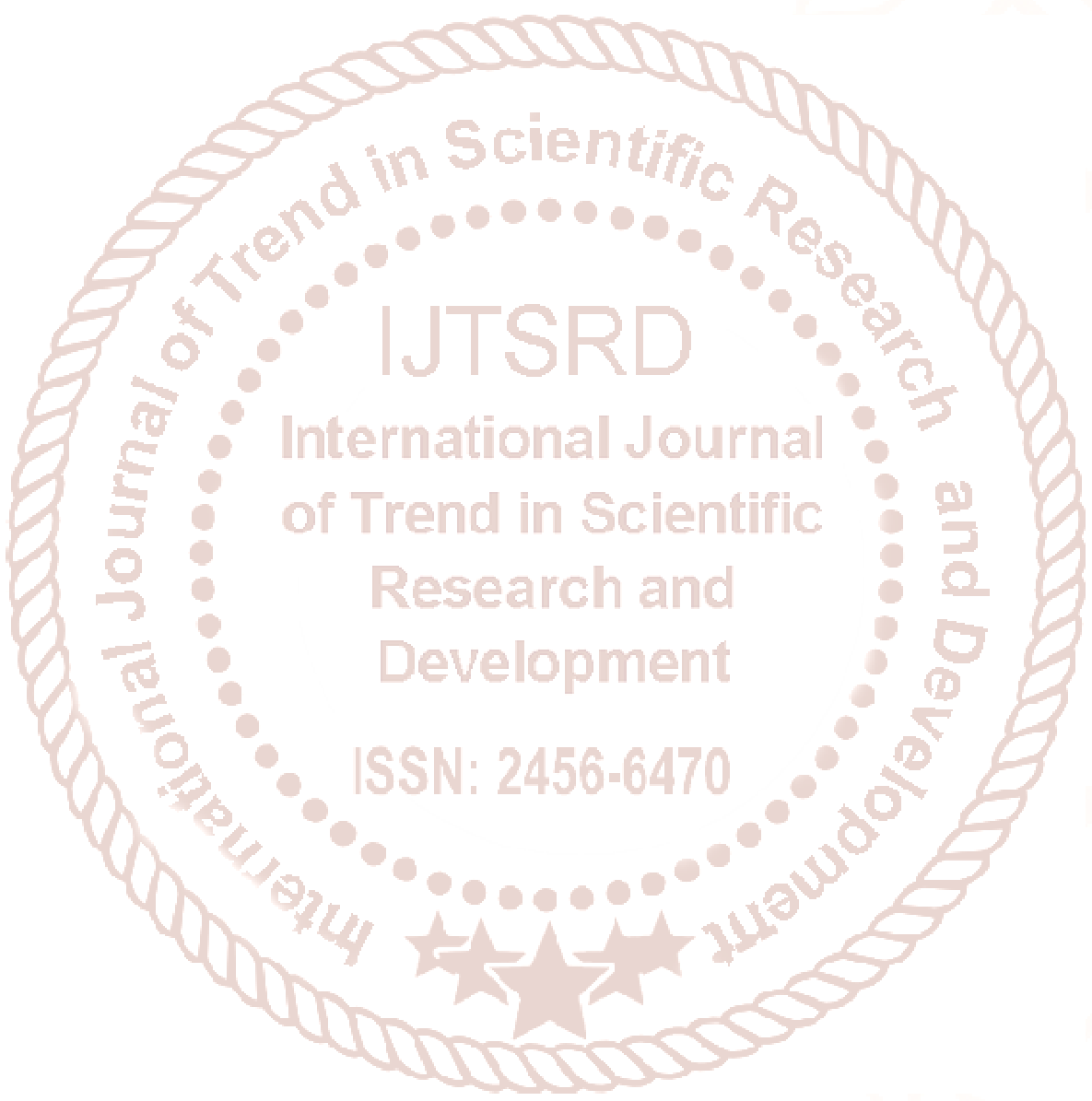

